\title{
Two Sample Bayes Prediction Scenario Under Right Censored Repairable System
}

\author{
Gyan Prakash ${ }^{1, *}$ and Sarvesh Kumar ${ }^{2}$ \\ ${ }^{1}$ Department Of Community Medicine, S. N. Medical College, Agra, India \\ ${ }_{2}^{2}$ Department Of Obs. \& Gyn., S. N. Medical College, Agra, India
}

Received 15 June 2013; Accepted 8 October 2013

Editor: Paulo Canas Rodrigues

\begin{abstract}
The objective of present article is to study the behavior of Bayes prediction length of interval under Two - Sample Bayes prediction scenario. A repairable system is considered here with assumption that the repair hazard rate increases monotonically as time parameter increases. Based on right item censoring criterion, the Bayes prediction length of interval and Highest Posterior Density (HPD) intervals have been obtain here for underlying model.
\end{abstract}

Keywords Repairable system, Right censoring, Predictive distribution, Two-Sample Bayes Predictive intervals, HPD intervals.

DOI: $10.19139 /$ soic.v1i1.17

\section{Introduction}

The prediction of future ordered observations shows, how long a sample of units might run until all fail in life testing. In many applications, technical systems or sub - systems have $k-o u t-o f-n$ structure, which has been extensively investigated in the literature. For such a system, the system consisting of $n$ components or subsystems, of which only $k$ need to be functioning and the system success $k$ is less than $n$. The $k-o u t-o f-n$ model is commonly used in reliability theory. In this model, the failure of any component of the system

\footnotetext{
${ }^{*}$ Correspondence to: Department Of Community Medicine, S. N. Medical College, Agra, India. Email: ggyanji@yahoo.com
}

ISSN 2310-5070 Copyright (c) 2013 International Academic Press 
does not influence the components still at work. It also finds wide applications in both industrial and military systems. These systems include the multi display system in cockpits, the multi-engine system in an airplane, and the multi-purpose system in a hydraulic control system.

The $(n-1)-$ out $-o f-n: G$ is a system that consists of $n$ components and works if and only if $(n-1)$ components among the $n$ work simultaneously. The system and each of its components can in only one of two states: working or failed. When a component fails, it kept under repair and other components stay in the working state with adjusted rates of failure. After repair, a component works as new and its actual lifetime is the same as initially. If the failed component is repair before another component fails, the $(n-1)$ components recover their initial lifetime. The lifetime and time of repair are independent.

For example, in the communications system with three transmitters, the average message load may be such that at least two transmitters must be operational at all times or critical messages may be lost. The transmission subsystem functions as a $2-o u t-o f-3: G$ system. The $k-o u t-o f-n$ system model may also represent systems with spares.

We consider here $1-$ out $-o f-n: G$, system which consists of $n$ components of the same kind with independent and identically distributed life - length. The system is observed under an inspection policy where inspection is made at the completion of a repair if it starts at the beginning of a repair. This leads us to a situation where separate observations on the unit's performance and on repair facility are not feasible. Thus, available records are the number of failures that occurred in a time interval between two repair epochs i.e., the time instant at which a repair completes. Recently, Prakash [7] studied some Bayes estimators for 1 - out - of $-n: G$, Repairable System.

James [5] studies reliabilities for consecutive $k-$ out - of $-n: F$ systems with component failures having $(k-1)$ step Markov dependence. The reliability function and failure rate of $k-o u t-o f-n$ system of components, with and without incorporating the environmental effect have discussed by Gupta [4]. Tian et al. [11] was discussed about the application of $k-o u t-o f-n$ structure in a very popular type of redundancy in fault tolerant systems, with wide applications in various types of systems. Gherda \& Boushaba [3] present an analysis of a repairable $(n-1)$ - out - of $-n: G$, system with failure and repair times arbitrarily distributed. Formulas for computing the minimal signature of $k-$ out $-o f-n$ systems based on their modules are derived by $\mathrm{Da}$ et al. [2]. Recently, an efficient method is proposed for exact reliability evaluation of $k-o u t-o f-n$ systems with identical components subject to phased mission 
requirements and imperfect fault coverage by Xing et al. [12].

Few of those who have been extensively studied recently about the predictive inference for the future observations are Raqab \& Madi [9], Nigm et al. [6], Sarhan \& Tadj [10], Ahmad et al. [1] and Prakash \& Singh [8].

The objective of present article is to predict the nature of future behavior of an observation when sufficient information of the past and present behavior of an event or an observation is known or given. We present here a Bayesian statistical analysis to predict the future order statistic from the considered repairable model under right censored (ordered) data. The One - Sided interval and interval of Central coverage under Two - Sample Bayes prediction scenario has been considered here. The model and prior distribution are described in Section 2. In Sections 3, the Bayes prediction lengths of interval are obtain. In next section the Highest Posterior Density (HPD) intervals are obtained. The last section includes an illustration of the proposed methods under a simulated dataset with a concluding subsection.

\section{Description of The Model Under Study}

A brief description about the considered $1-o u t-o f-n: G$ repairable system is based on following assumptions:

The system consists with $n$ units and having a repair facility. Initially one unit starts operating and the remaining $(n-1)$ are kept as inactive standbys. As soon as a unit fails, it goes for repair and a standby unit is put on the operation. The repair policy is based on First Come First Serves (FCFS), it is always open, and the repairs are perfect with negligible switch over time.

The failure time distribution of the online units and repair time distribution of units under repair are assume general, independent of each other and both are increasing failure rate (IFR) distributions. The state of the system is defined by the number of non - operative units in the system at the time $t(>0)$. Further, the state $n$ is called the down state of the system.

The system is observed under an inspection policy where inspection is made at the completion of a repair provided that it starts at the beginning of a repair. This leads us to a situation where separate observations on the unit's performance and on repair facility are not feasible. Thus, available records are the number of failures that occurred in the time interval between two repair epochs i.e., the time instant at which a repair completes. 
Let, $X(t)=i ; i=0,1,2, \ldots, n$ be the number of non - operative units at time $t$. We put a system on test and the system is observed continuously until $n$ repairs are over. Let the system be in state $i(=1,2, \ldots, n-1)$ initially whenever a first repair starts and states of the process are recorded at the completion of each repair. The hazard rate $\rho_{i k}(t)$ of the system in the state $k$ if it was in the state $i$ is given as

$$
\rho_{i k}(t)=\frac{n_{i k}}{u+1} t^{v} ; u+1>n_{i k}, v>0 .
$$

Here, $u$ be the number of units failed when system transits from state $i$ to state $k$. It is also noted that the hazard rate decreases with $u$ increases. Hazard rate after re - parameterization

$$
\rho(t)=\theta t^{v} ; \theta=\frac{n_{i k}}{u+1} .
$$

In life testing, fatigue failures and other kinds of destructive test situations, the observations usually occurred in ordered manner such a way that weakest items failed first and then the second one and so on. Let us suppose that $n$ items are put to test under the considered repairable model without replacement and only $k$ items are fully measured, while the remaining $(n-k)$ items are censored. These $(n-k)$ censored lifetimes will be ordered separately. This is called as right item censoring scheme. The $n$ ordered items $x_{(1)}, x_{(2)}, \ldots, x_{(k)}, \ldots, x_{(n)}$ are put to test under the model without replacement and in which $k$ orders items are fully measured.

Using the right censoring criterion, the likelihood function is thus obtained as

$$
\begin{gathered}
L\left(x_{(1)}, x_{(2)}, \ldots, x_{(k)} \mid \theta\right)=\left(\prod_{i=1}^{k} f\left(x_{(i)} ; \theta\right)\right) \cdot\left(\prod_{i=k+1}^{n} \frac{f\left(x_{(i)} ; \theta\right)}{\rho\left(x_{(i)}\right)}\right) \\
=\theta^{k}\left(\prod_{i=1}^{k} x_{(i)}^{v}\right) e^{-\theta T_{1}} ; T_{1}=\frac{1}{v+1} \sum_{i=1}^{n} x_{(i)}^{v+1} .
\end{gathered}
$$

Here, $T_{1}$ is a sufficient statistic for parameter $\theta$ and the maximum likelihood (ML) estimate of the parameter $\theta$ is

$$
\hat{\theta}_{M L}=k T_{1}^{-1} .
$$

From a Bayesian viewpoint, there is clearly no way in which one can say that one prior is better than other. It is more frequently the case that, we select to restrict attention to a given flexible family of priors, and we choose one from that family, which seems to match best with our personal beliefs. One of best choices for selecting the prior distribution is conjugate prior. We consider here Gamma density as a natural family of conjugate prior for parameter $\theta$ and is given as

$$
\pi(\theta)=\frac{\beta^{\alpha}}{\Gamma(\alpha)} \theta^{\alpha-1} \exp (-\beta \theta) ; \alpha>0, \beta>0, \theta>0 .
$$




\section{Bayes Prediction Intervals Under Two - Sample Scenario}

Since $x_{(1)}, x_{(2)}, \ldots, x_{(k)}$ be the first $k$ fully measured items are observed from, a sample of size $n$ of the considered model. Let $y_{(1)}, y_{(2)}, \ldots, y_{(m)}$ is the second (unobserved) items censored data of size $m$ drawn independently from sample of size $N$ of the same model, then first sample is referred as informative (past) sample, while the second one is referred as the future sample. Based on an informative item failure censored sample, our aim is to predict the $j^{\text {th }}$ order statistic in the future sample. This technique is called as Two-Sample Bayes prediction technique.

Now, the Bayes predicative density of the future observation $Y$ is denoted by $h(y \mid \underline{x})$ and obtained by simplifying

$$
h(y \mid \underline{x})=\int_{\theta} f(y ; \theta) \cdot \pi(\theta \mid \underline{x}) d \theta .
$$

Here, $\pi(\theta \mid \underline{x})$ be the posterior density of the parameter $\theta$ and obtained with respect to the prior density given in equation (4). Hence, the Bayes predictive density is given by

$$
h(y \mid \underline{x})=(\alpha+k)\left(T_{1}+\beta\right)^{\alpha+k} y^{v}\left(T_{1}+\beta+\frac{y^{v+1}}{v+1}\right)^{-\alpha-k-1} .
$$

Basically, predictive density $h(y \mid \underline{x})$ expresses the plausibility of $y$ given $x$ and $\pi(\theta)$. Based on Bayes predictive density of future observation $Y$, the cumulative density function is thus obtained as

$$
G(y \mid \underline{x})=P(Y \leq y)=1-\left(1+\frac{y^{v+1}}{(v+1)\left(T_{1}+\beta\right)}\right)^{-\alpha-k} .
$$

If we consider $m$ future observations, then probability density function of the $j^{\text {th }}$ ordered future observation is

$$
\phi\left(Y_{j}\right)=j\left(\begin{array}{l}
m \\
j
\end{array}\right)(G(y \mid \underline{x}))^{j-1}(1-(G(y \mid \underline{x})))^{m-j} h(y \mid \underline{x}) .
$$

Now, the Bayes prediction limits with coverage $(1-\varepsilon)$ is defined for a future random variable $Y$ as

$$
P\left(l_{1} \leq Y \leq l_{2}\right)=1-\varepsilon,
$$

where $l_{1}$ and $l_{2}$ are lower and upper Bayes prediction limits for random variable $Y$, and $(1-\varepsilon)$ is called the confidence prediction coefficient.

To find the prediction limits for $Y_{j}$, the $j^{t h}$ smallest of a set of $m$ future observations under probability density function (8), we choose $l_{1 j}$ and $l_{2 j}$ such as,

$$
P\left(l_{1 j} \leq Y_{j} \leq l_{2 j}\right)=1-\varepsilon \text {. }
$$




\subsection{One - Sided Bayes Prediction Limits}

The expressions for one - sided $100(1-\varepsilon) \%$ Bayes prediction limits of the $j^{\text {th }}$ future observations are obtain by solving

$$
j\left(\begin{array}{c}
m \\
j
\end{array}\right) \int_{0}^{l_{1}} Z^{j-1}(1-Z)^{m-j} d Z=\frac{\varepsilon}{2}
$$

and

$$
j\left(\begin{array}{c}
m \\
j
\end{array}\right) \int_{0}^{l_{2}} Z^{j-1}(1-Z)^{m-j} d Z=1-\frac{\varepsilon}{2},
$$

where $l_{i}=1-\left(1+\frac{l_{i j}^{v+1}}{(v+1)\left(T_{1}+\beta\right)}\right)^{-\alpha-k}$ and $i=1,2 ; j=1,2, \ldots, m$.

The Bayes prediction limits for first future observation are obtained, by solving (9), for $j=1$ as

$$
l_{11}=\left\{(v+1)\left(T_{1}+\beta\right)\left(\left(\left(1-\frac{\varepsilon}{2}\right)^{1 / m}\right)^{-1 /(\alpha+k)}-1\right)\right\}^{\frac{1}{v+1}}
$$

and

$$
l_{21}=\left\{(v+1)\left(T_{1}+\beta\right)\left(\left(\left(\frac{\varepsilon}{2}\right)^{1 / m}\right)^{-1 /(\alpha+k)}-1\right)\right\}^{\frac{1}{v+1}} .
$$

The Bayes prediction length of intervals for the smallest (first one) future observation is

$$
I_{1}=l_{21}-l_{11} .
$$

Similarly, the Bayes prediction limits for last future observation are obtained, by solving (9), for $j=m$ as

$$
l_{1 m}=\left\{(v+1)\left(T_{1}+\beta\right)\left(\left(1-\left(\frac{\varepsilon}{2}\right)^{1 / m}\right)^{-1 /(\alpha+k)}-1\right)\right\}^{1 /(v+1)}
$$

and

$$
l_{2 m}=\left\{(v+1)\left(T_{1}+\beta\right)\left(\left(1-\left(1-\frac{\varepsilon}{2}\right)^{1 / m}\right)^{-1 /(\alpha+k)}-1\right)\right\}^{1 /(v+1)} .
$$

Also, the Bayes prediction length of intervals for largest (last one) future observation is given as

$$
I_{m}=l_{2 m}-l_{1 m}
$$




\subsection{The Central Coverage Bayes Prediction Limits}

The central coverage Bayes prediction limits are obtain by solving following equations for the $j^{t h}$ future observations

$$
j\left(\begin{array}{c}
m \\
j
\end{array}\right) \int_{0}^{l_{1}} Z^{j-1}(1-Z)^{m-j} d Z=\frac{1-\varepsilon}{2}
$$

and

$$
j\left(\begin{array}{c}
m \\
j
\end{array}\right) \int_{0}^{l_{2}} Z^{j-1}(1-Z)^{m-j} d Z=\frac{1+\varepsilon}{2}
$$

For smallest future observations, the central coverage Bayes prediction limits are obtain as

$$
l_{11 C}=\left\{(v+1)\left(T_{1}+\beta\right)\left(\omega_{1}^{-1 /(\alpha+k)}-1\right)\right\}^{1 /(v+1)} ; \omega_{1}=\left(\frac{1+\varepsilon}{2}\right)^{1 / m}
$$

and

$$
l_{21 C}=\left\{(v+1)\left(T_{1}+\beta\right)\left(\omega_{2}^{-1 /(\alpha+k)}-1\right)\right\}^{1 /(v+1)} ; \omega_{2}=\left(\frac{1-\varepsilon}{2}\right)^{1 / m} .
$$

Similarly, the central coverage Bayes prediction limits for the largest future observations are

$$
l_{1 m C}=\left\{(v+1)\left(T_{1}+\beta\right)\left(\left(1-\omega_{2}\right)^{-1 /(\alpha+k)}-1\right)\right\}^{1 /(v+1)}
$$

and

$$
l_{2 m C}=\left\{(v+1)\left(T_{1}+\beta\right)\left(\left(1-\omega_{1}\right)^{-1 /(\alpha+k)}-1\right)\right\}^{1 /(v+1)} .
$$

Hence, the length of Bayes prediction intervals under the central coverage are given respectively as

$$
I_{1 C}=l_{21 C}-l_{11 C}
$$

and

$$
I_{m C}=l_{2 m C}-l_{1 m C}
$$

\section{Highest Posterior Density (HPD) Intervals}

In this section, our objective is to provide highest posterior density (HPD) interval for the unknown parameter $\theta$ of the considered model under the right censoring criterion. Since, the posterior density $\pi(\theta \mid \underline{x})$ corresponding to the parameter $\theta$ is unimodel. Thus, $100(1-\varepsilon) \%$ HPD interval $\left[H_{1}, H_{2}\right.$ [ for the parameter $\theta$ must 
satisfy the following equations simultaneously.

$$
\int_{H_{1}}^{H_{2}} \pi(\theta \mid \underline{x}) d \theta=1-\varepsilon
$$

and

$$
\pi\left(H_{1} \mid \underline{x}\right)=\pi\left(H_{2} \mid \underline{x}\right) .
$$

Now, the expression (15) \& (16) rewritten as

$$
\begin{gathered}
\int_{H_{1}\left(T_{1}+\beta\right)}^{H_{2}\left(T_{1}+\beta\right)} \frac{1}{\Gamma(\alpha+k)} e^{-z} z^{\alpha+k-1} d z=1-\varepsilon \\
\Rightarrow \frac{1}{\Gamma(\alpha+k)}\left[\gamma\left\{(\alpha+k), H_{2}\left(T_{1}+\beta\right)\right\}-\gamma\left\{(\alpha+k), H_{1}\left(T_{1}+\beta\right)\right\}\right]=1-\varepsilon
\end{gathered}
$$

and

$$
\left(\frac{H_{2}}{H_{1}}\right)^{\alpha+k-1}=e^{-\left(H_{1}-H_{2}\right)\left(T_{1}+\beta\right)} .
$$

Solve simultaneously the equations (17) and (18) to obtain the highest posterior density limits $H_{1}$ and $H_{2}$.

\section{Numerical Analysis}

We illustrate the procedure by presenting a complete analysis under a simulated data set. A data set of 10,000 random samples has been drawn from the considered model for a different set of parametric values. The considered value of the sample size $n$ is 15 with censored sample size $k(=4,8,12)$ with numerical values of the parameter $\theta(=4,6,8)$. The constant $v$ is considered to be fixed at unity. The values of the prior parameters $(\alpha, \beta)$ are taken in the combination of $(\alpha, \beta)=$ $(0.25,0.50),(1,1),(4,2),(9,3)$. The values of the prior parameters $\alpha$ and $\beta$ meets the criterion that the prior variance should be unity.

\subsection{Bayes Prediction Length of Intervals}

Using above considered parametric values, the Bayes prediction length of intervals have been calculated and presented them in the Tables $1-2$ for the size of future observation $m(=4)$ with confidence level $\varepsilon=99 \%, 95 \%, 90 \%$.

It is observed from Table 1 that, the Bayes prediction lengths of intervals tend to closer (wider) as $\varepsilon(k)$ increases when other parametric values are considered to be fixed. The prediction length of intervals expended also when prior parameters $(\alpha, \beta)$ or, parameter $\theta$ increases for other fixed parametric values. 
Table 1. One - Sided Bayes Prediction Length of Intervals

\begin{tabular}{|c|c|c|c|c|c|c|c|}
\hline \multicolumn{2}{|r|}{$n=15$} & \multicolumn{3}{|c|}{ First Future Observation } & \multicolumn{3}{|c|}{ Last Future Observation } \\
\hline \multirow[t]{2}{*}{\begin{tabular}{l|l}
$k$ & $\theta$ \\
\end{tabular}} & $(\alpha, \beta) \Downarrow \varepsilon \Rightarrow$ & $99 \%$ & $95 \%$ & $90 \%$ & $99 \%$ & $95 \%$ & $90 \%$ \\
\hline & $0.25,0.50$ & 0.5722 & 0.4237 & 0.3954 & 0.6834 & 0.5258 & 0.5000 \\
\hline \multirow[t]{3}{*}{0404} & 01,01 & 0.8476 & 0.6238 & 0.5804 & 1.0147 & 0.7806 & 0.7369 \\
\hline & 04,02 & 1.0522 & 0.7761 & 0.7217 & 1.2588 & 0.9697 & 0.9186 \\
\hline & 09,03 & 1.3737 & 1.0147 & 0.9411 & 1.6471 & 1.2657 & 1.1977 \\
\hline \multirow{4}{*}{0408} & $0.25,0.50$ & 0.6199 & 0.4585 & 0.4258 & 0.7447 & 0.5734 & 0.5421 \\
\hline & 01,01 & 0.8876 & 0.6561 & 0.6092 & 1.0641 & 0.8193 & 0.7746 \\
\hline & 04,02 & 1.0914 & 0.8069 & 0.7475 & 1.3080 & 1.0068 & 0.9518 \\
\hline & 09,03 & 1.4138 & 1.0444 & 0.9677 & 1.6934 & 1.3034 & 1.2318 \\
\hline \multirow{4}{*}{0804} & $0.25,0.50$ & 0.8163 & 0.6030 & 0.5600 & 0.9340 & 0.7258 & 0.6903 \\
\hline & 01,01 & 1.2178 & 0.8950 & 0.8268 & 1.4174 & 1.0733 & 1.0218 \\
\hline & 04,02 & 1.5202 & 1.1117 & 1.0388 & 1.7614 & 1.3411 & 1.2706 \\
\hline & 09,03 & 1.9927 & 1.4520 & 1.3569 & 2.2954 & 1.7465 & 1.6588 \\
\hline \multirow{4}{*}{0808} & $0.25,0.50$ & 0.8975 & 0.6586 & 0.6108 & 1.0403 & 0.7918 & 0.7500 \\
\hline & 01,01 & 1.2906 & 0.9414 & 0.8746 & 1.4876 & 1.1296 & 1.0717 \\
\hline & 04,02 & 1.5820 & 1.1549 & 1.0753 & 1.8266 & 1.3886 & 1.3165 \\
\hline & 09,03 & 2.0523 & 1.4976 & 1.3932 & 2.3640 & 1.7974 & 1.7055 \\
\hline \multirow{4}{*}{1204} & $0.25,0.50$ & 1.6259 & 1.1776 & 1.0542 & 1.7312 & 1.3019 & 1.1876 \\
\hline & 01,01 & 2.3035 & 1.6670 & 1.4925 & 2.4512 & 1.8424 & 1.6810 \\
\hline & 04,02 & 2.8222 & 2.0418 & 1.8275 & 3.0037 & 2.2585 & 2.0595 \\
\hline & 09,03 & 3.6441 & 2.6367 & 2.3605 & 3.8788 & 2.9158 & 2.6590 \\
\hline \multirow{4}{*}{1208} & $0.25,0.50$ & 1.6561 & 1.1984 & 1.0726 & 1.7628 & 1.3250 & 1.2084 \\
\hline & 01,01 & 2.3419 & 1.6949 & 1.5171 & 2.4930 & 1.8741 & 1.7091 \\
\hline & 04,02 & 2.8684 & 2.0758 & 1.8580 & 3.0535 & 2.2953 & 2.0934 \\
\hline & 09,03 & 3.7033 & 2.6799 & 2.3987 & 3.9420 & 2.9633 & 2.7024 \\
\hline
\end{tabular}

The central coverage Bayes prediction lengths of intervals are presented in Table 2. It is noted that the Bayes prediction length of intervals tend to wider as $k$ (for $k=4,8$ only) increases for small $(\alpha, \beta)$. Other, behaviors are similar as seen in case of one - sided Bayes prediction length of interval. It is also seen that the central coverage prediction length of interval closer to one - sided prediction length of interval for all considered parametric values. It is further noted that when confidence level decreases length of intervals also decreases. This shows that the lengths of intervals are robust.

Further, the length of intervals tend to wider when $m$ increases. This is a natural, since the prediction of future order statistic that is far away from the last observed value and has less accuracy than that of other future order statistics. 
Table 2. Central Coverage Bayes Prediction Length of Intervals

\begin{tabular}{|c|c|c|c|c|c|c|c|}
\hline \multicolumn{2}{|r|}{$n=15$} & \multicolumn{3}{|c|}{ First Future Observation } & \multicolumn{3}{|c|}{ Last Future Observation } \\
\hline \multirow[t]{2}{*}{\begin{tabular}{l|l}
$k$ & $\theta$ \\
\end{tabular}} & $\theta \mid(\alpha, \beta) \Downarrow \varepsilon \Rightarrow$ & $99 \%$ & $95 \%$ & $90 \%$ & $99 \%$ & $95 \%$ & $90 \%$ \\
\hline & $0.25,0.50$ & 0.5088 & 0.3767 & 0.3515 & 0.6076 & 0.4675 & 0.4445 \\
\hline \multirow[t]{3}{*}{0404} & 01,01 & 0.7535 & 0.5546 & 0.5160 & 0.9022 & 0.6940 & 0.6551 \\
\hline & 04,02 & 0.9355 & 0.6900 & 0.6416 & 1.1192 & 0.8621 & 0.8167 \\
\hline & 09,03 & 1.2213 & 0.9022 & 0.8367 & 1.4644 & 1.1252 & 1.0648 \\
\hline \multirow{4}{*}{0408} & $0.25,0.50$ & 0.5453 & 0.4033 & 0.3745 & 0.6551 & 0.5044 & 0.4769 \\
\hline & 01,01 & 0.7808 & 0.5772 & 0.5358 & 0.9360 & 0.7207 & 0.6814 \\
\hline & 04,02 & 0.9601 & 0.7098 & 0.6575 & 1.1506 & 0.8856 & 0.8373 \\
\hline & 09,03 & 1.2437 & 0.9187 & 0.8512 & 1.4896 & 1.1465 & 1.0835 \\
\hline \multirow{4}{*}{080} & $0.25,0.50$ & 0.7258 & 0.5361 & 0.4979 & 0.8303 & 0.6453 & 0.6137 \\
\hline & 01,01 & 1.0827 & 0.7957 & 0.7351 & 1.2601 & 0.9543 & 0.9084 \\
\hline & 04,02 & 1.3516 & 0.9884 & 0.9235 & 1.5660 & 1.1923 & 1.1297 \\
\hline & 09,03 & 1.7717 & 1.2909 & 1.2064 & 2.0407 & 1.5528 & 1.4748 \\
\hline \multirow{4}{*}{0808} & $0.25,0.50$ & 0.7895 & 0.5793 & 0.5373 & 0.9151 & 0.6965 & 0.6597 \\
\hline & 01,01 & 1.1353 & 0.8281 & 0.7694 & 1.3086 & 0.9937 & 0.9427 \\
\hline & 04,02 & 1.3916 & 1.0159 & 0.9459 & 1.6068 & 1.2215 & 1.1581 \\
\hline & 09,03 & 1.8054 & 1.3173 & 1.2255 & 2.0795 & 1.5811 & 1.5003 \\
\hline \multirow{4}{*}{1204} & $0.25,0.50$ & 1.4456 & 1.0470 & 0.9373 & 1.5391 & 1.1574 & 1.0559 \\
\hline & 01,01 & 2.0479 & 1.4820 & 1.3269 & 2.1793 & 1.6380 & 1.4945 \\
\hline & 04,02 & 2.5091 & 1.8153 & 1.6248 & 2.6705 & 2.0080 & 1.8310 \\
\hline & 09,03 & 3.2399 & 2.3442 & 2.0986 & 3.4485 & 2.5923 & 2.3640 \\
\hline \multirow{4}{*}{1208} & $0.25,0.50$ & 1.4568 & 1.0541 & 0.9435 & 1.5506 & 1.1656 & 1.0630 \\
\hline & 01,01 & 2.0601 & 1.4909 & 1.3345 & 2.1930 & 1.6486 & 1.5034 \\
\hline & 04,02 & 2.5232 & 1.8260 & 1.6344 & 2.6860 & 2.0190 & 1.8414 \\
\hline & 09,03 & 3.2576 & 2.3574 & 2.1101 & 3.4676 & 2.6067 & 2.3772 \\
\hline
\end{tabular}

\subsection{HPD Intervals}

With above considered parametric values, the HPD limits have been obtain and presented them in Table 3 for different coverage probability level $\varepsilon=$ $99 \%, 95 \%, 90 \%$.

It is observed from table that the HPD length of interval becomes narrower as prior parameter $(\alpha, \beta)$ or parameter $\theta$ increases. Similar trend also has been seen when censored sample size $k$ increase when other parametric values are considered to be fixed. Further, the HPD length of interval becomes wider as the coverage probability $\varepsilon$ increases. 
Table 3. Highest Posterior Density (HPD) Limits

\begin{tabular}{|c|c|c|c|c|c|c|c|}
\hline \multicolumn{2}{|r|}{$n=15$} & \multicolumn{3}{|c|}{$H_{1}$} & \multicolumn{3}{|c|}{$\mathrm{H}_{2}$} \\
\hline \begin{tabular}{l|l|}
$k$ & $\theta$
\end{tabular} & $(\alpha, \beta) \Downarrow \varepsilon \Rightarrow$ & $99 \%$ & $95 \%$ & $90 \%$ & $99 \%$ & $95 \%$ & $90 \%$ \\
\hline \multirow{4}{*}{0404} & $0.25,0.50$ & 0.8774 & 0.8658 & 0.8543 & 2.1336 & 2.0649 & 1.9983 \\
\hline & 01,01 & 0.8613 & 0.8500 & 0.83 & .0410 & 1.9753 & 1.9117 \\
\hline & 04,02 & 0.8490 & 0.8378 & 0.8267 & 1.9670 & 1.9037 & 1.8424 \\
\hline & 09,03 & 0.7969 & 0.7863 & 0.7759 & 8464 & 1.7870 & 1.7295 \\
\hline \multirow{4}{*}{0408} & $0.25,0.50$ & 0.9995 & 0.9863 & 0.9733 & 1.6215 & 1.5079 & 1.4500 \\
\hline & 01,01 & 0.9921 & 0.9789 & 0.9660 & 1.6030 & 1.4944 & 1.4401 \\
\hline & 04,02 & 0.9872 & 0.9741 & 0. & 1.5882 & 1.4820 & 1.4290 \\
\hline & 09,03 & 0.9366 & 0.9241 & 0.9119 & 1.5067 & 1.4060 & 1.3557 \\
\hline \multirow{4}{*}{0804} & $0.25,0.50$ & 0.9953 & 0.9823 & 0.9692 & 2.1053 & 2.0375 & 1.9718 \\
\hline & 01,01 & 0.9772 & 0.9642 & 0.9515 & 2.0140 & 1.9491 & 1.8864 \\
\hline & 04,02 & 0.9631 & 0.9504 & 0.9 & 10 & 1.8785 & 1.8179 \\
\hline & 09,03 & 0.9042 & 0.8921 & 0.8803 & 1.8220 & 1.7633 & 1.7065 \\
\hline \multirow{4}{*}{0808} & $0.25,0.50$ & 1.1518 & 1.1365 & 1.1215 & 1.5694 & 1.4595 & 1.4034 \\
\hline & 01,01 & 1.1433 & 1.1280 & 1.1131 & 1.5515 & 1.4464 & 1.3938 \\
\hline & 04,02 & 1.1375 & 1.1224 & 1.10 & 1.5372 & 1.4344 & 1.3831 \\
\hline & 09,03 & 1.0793 & 1.0649 & 1.0509 & 1.4583 & 1.3609 & 1.3121 \\
\hline \multirow{4}{*}{1204} & $0.25,0.50$ & 1.0052 & 0.9920 & 0.9788 & 2.0634 & 1.9969 & 1.9326 \\
\hline & 01,01 & 0.9870 & 0.9739 & 0.96 & 39 & 1.9104 & 1.8489 \\
\hline & 04,02 & 0.9728 & 0.9599 & 0.9472 & 1.9023 & 1.8411 & 1.7818 \\
\hline & 09,03 & 0.9132 & 0.9009 & 0.8891 & 1.7857 & 1.7282 & 1.6726 \\
\hline \multirow{4}{*}{1208} & $0.25,0.50$ & 1.1623 & 1.1469 & 1.1317 & 1.5178 & 1.4114 & 1.3573 \\
\hline & 01,01 & 1.1537 & 1.1382 & 1.1232 & 1.5004 & 1.3987 & 1.3479 \\
\hline & 04,02 & 1.1479 & 1.1327 & 1.11 & 1.4866 & 1.3873 & 1.3375 \\
\hline & 09,03 & 1.0890 & 1.0746 & 1.0604 & 1.4103 & 1.3161 & 1.2689 \\
\hline
\end{tabular}

Remark: In the case when the censored sample size $k(=15)$, the censoring criterion is reduces to the complete sample size criterion and hence all the result are valid for complete sample case.

\section{Conclusion}

In present article, we study the behavior of Bayes prediction length of interval and HPD intervals based on Two - Sample Bayes prediction scenario for a repairable system. The system is considered here with assumption that repair hazard rate increases monotonically as time parameter increases. 
Based on simulation study, it is observed that the Bayes prediction lengths of intervals tend to be closer as coverage probability increases. Similar properties also have been seen in case of Central coverage Bayes prediction length of interval. It is also seen that the Central coverage prediction length of interval closer to the One - Sided prediction length of interval for all considered parametric values. When confidence level decreases the length of intervals also decreases. This shows that the lengths of intervals are robust. It is also noted that the Highest Posterior Density (HPD) length of interval becomes wider as the coverage probability $\varepsilon$ increases.

\section{REFERENCES}

1. A. A. Ahmad, Z. R. Mohammad, \& T. M. Mohamed, Bayesian prediction intervals for the future order statistics from the generalized Exponential distribution. Journal of Iranian Statistical Society, 6 (1): 17-30, 2007.

2. G. Da, L.Xia, \& T. Hu, On computing signatures of $k$-out-of- $n$ systems consisting of modules. Methodology and Computing in Applied Probability, 2012: 1-11, 2012.

3. M. Gherda,\& M. Boushaba, Analysis of a repairable (n-1)-out-of- $n$ : $G$, system with failure and repair times arbitrarily distributed. American Journal of Mathematics and Statistics, 1 (1): 1-7, 2011.

4. R. C. Gupta, Reliability of a $k$-out-of- $n$ system of components sharing a common environment. Applied Mathematics Letters, 15 (7): 837-844, 2002.

5. F. C. James, Reliabilities are studied for consecutive $k$-out-of- $n: F$ systems with component failures having ( $k-1)$-step Markov dependence. IEEE Transactions on Reliability, 35 (5): 602606, 1986.

6. A. M. Nigm, E. K. AL - Hussaini, \& Z. F. Jaheen, Bayesian one-sample prediction of future observations under Pareto distribution. Statistics, 37 (6): 527-536, 2003.

7. G. Prakash, Some bayes estimators for the repairable system. Model Assisted Statistics and Applications, 6 (1): 57-62, 2011.

8. G. Prakash,\& D. C. Singh, Bayes prediction intervals for the Pareto model. Journal of Probability and Statistical Science, 11 (1): 109-122, 2013.

9. M. Z. Raqab, \& M. T. Madi, Bayesian prediction of the total time on test using doubly censored Rayleigh data. Journal of Statistical Computational and Simulation, 72: 781-789, 2002.

10. A. M. Sarhan, \& L. Tadj, Parameters estimation of a repairable system. Applied Mathematics and Computation, 138 (2-3): 217-226, 2003.

11. Z. Tian, W. Li, \& J. M. Zuo, Modeling and reliability evaluation of multi-state $k$-out-of- $n$ systems. Recent Advances in Reliability and Quality in Design, 2008: 31-56, 2008.

12. L. Xing, S. V. Amari, \& C. Wang, Reliability of $k$-out-of- $n$ systems with phased - mission requirements and imperfect fault coverage. Reliability Engineering and System Safety, 103: 45-50, 2012. 